\title{
First correlated measurements of the shape and light scattering properties of cloud particles using the new Particle Habit Imaging and Polar Scattering (PHIPS) probe
}

\author{
A. Abdelmonem ${ }^{1}$, M. Schnaiter ${ }^{1}$, P. Amsler ${ }^{2}$, E. Hesse ${ }^{3}$, J. Meyer ${ }^{4}$, and T. Leisner ${ }^{1}$ \\ ${ }^{1}$ Institute for Meteorology and Climate Research, Karlsruhe Institute of Technology, Karlsruhe, Germany \\ ${ }^{2}$ ETH Zurich Institute for Atmospheric and Climate Science, Universitaetstrasse 16, 8092 Zurich, Switzerland \\ ${ }^{3}$ Centre for Atmospheric and Instrumentation Research, University of Hertfordshire, Hatfield, AL10 9AB, UK \\ ${ }^{4}$ Jülich Research Center, ICG-1, Jülich, Germany
}

Received: 6 April 2011 - Published in Atmos. Meas. Tech. Discuss.: 17 May 2011

Revised: 26 September 2011 - Accepted: 29 September 2011 - Published: 12 October 2011

\begin{abstract}
Studying the radiative impact of cirrus clouds requires knowledge of the relationship between their microphysics and the single scattering properties of cloud particles. Usually, this relationship is obtained by modeling the optical scattering properties from in situ measurements of ice crystal size distributions. The measured size distribution and the assumed particle shape might be erroneous in case of non-spherical ice particles. We present here a novel optical sensor (the Particle Habit Imaging and Polar Scattering probe, PHIPS) designed to measure simultaneously the 3-D morphology and the corresponding optical and microphysical parameters of individual cloud particles. Clouds containing particles ranging from a few micrometers to about $800 \mu \mathrm{m}$ diameter in size can be characterized systematically with an optical resolution power of $2 \mu \mathrm{m}$ and polar scattering resolution of $1^{\circ}$ for forward scattering directions (from $1^{\circ}$ to $10^{\circ}$ ) and $8^{\circ}$ for side and backscattering directions (from $18^{\circ}$ to $170^{\circ}$ ). The maximum acquisition rates for scattering phase functions and images are $262 \mathrm{KHz}$ and $10 \mathrm{~Hz}$, respectively. Some preliminary results collected in two ice cloud campaigns conducted in the AIDA cloud simulation chamber are presented. PHIPS showed reliability in operation and produced size distributions and images comparable to those given by other certified cloud particles instruments. A 3-D model of a hexagonal ice plate is constructed and the corresponding scattering phase function is compared to that
\end{abstract}

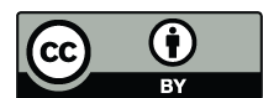

Correspondence to: A. Abdelmonem (ahmed.abdelmonem@kit.edu) modeled using the Ray Tracing with Diffraction on Facets (RTDF) program. PHIPS is a highly promising novel airborne optical sensor for studying the radiative impact of cirrus clouds and correlating the particle habit-scattering properties which will serve as a reference for other single, or multi-independent, measurement instruments.

\section{Introduction}

Better understanding of the radiative impact of cirrus clouds and a possible change of this impact by human activity requires knowledge of the link between the cirrus cloud microphysics and the single scattering properties of the cloud particles which are used in radiative transfer models. Usually, this link is created by using in situ measurements of the ice crystal size distributions as input for optical scattering models like the Mie Theory to calculate the scattering properties. This gives rise to the following problems: (i) the size distribution measured by scattering spectrometers might be erroneous in case of non-spherical ice particles. (ii) The shape assumed in the Mie optical model is certainly different from the real ice particle habit, which results in wrong optical parameters of the whole particle ensemble. Although sophisticated optical models for the computation of the scattering properties of irregularly shaped ice particles have been developed over the last 20 years (e.g. Takano and Liu, 1995; Yang et al., 2000), there are still discrepancies between the cloud radiative properties derived by spaceborne remote sensing and those derived from in situ microphysical

Published by Copernicus Publications on behalf of the European Geosciences Union. 
properties using sophisticated libraries of single scattering properties (Baum et al., 2010). Therefore, the microphysical and optical properties of ice crystals have to be measured by an in situ instrument.

Interpretations of microphysical parameters of cloud particles are based on assumptions on the particle shape. For example, the modeling approach to retrieve ice particle parameters used by Shcherbakov et al. (2006a,b) assumed crystals to be hexagonal columns/plates. The airborne polar nephelometer (PN) by Gayet et al. (2001) measures the scattering function of the ice particles and was used in conjunction with results from an imaging probe (CPI) (Lawson et al., 2001) to investigate the impact of the ice crystal habits on the radiative properties of cirrus clouds. This could be done only in a statistical approach with assumptions made regarding the particle shape within an ensemble of randomly oriented particles. In their case study during the ASTAR airborne campaign in the southeast of the Svalbard Archipelago, Jourdan et al. (2010) used a principle component analysis (PCA) of the scattering phase function measured by the PN together with particle habits revealed by CPI to link the microphysical and shape properties of cloud particles to their single scattering properties. This link required a clustering of the collected results according to optical parameters of particles and is only true for particles with sizes larger than $50 \mu \mathrm{m}$, as the optical contribution of small particles could not be determined directly in a mixed-phase cloud. A direct link between the particle habit and the corresponding microphysical parameters is still lacking, at least for complex shapes.

The PHIPS instrument combines stereo imaging of individual cloud particles with simultaneous measurement of the polar scattering function of the same particle. The prototype particle imager of PHIPS, as described by Schön et al. (2011), has now been completed by a second identical imaging unit to image the same particle under an angular viewing distance of $60^{\circ}$ (at $30^{\circ}$ and $-30^{\circ}$ from the laser beam) and by a polar nephelometer for the simultaneous measurement of the single particle scattering phase function. Note that the viewing angle with respect to the laser beam slightly differs from that $\left(29^{\circ}\right)$ used in the prototype particle imager of PHIPS. PHIPS is the first step towards PHIPSHALO which is one of the novel instruments that are currently under development for the new German research aircraft HALO. The resolution power of both microscope units is about $2 \mu \mathrm{m}$. The dual imaging configuration facilitates a 3-D morphology impression of the ice crystals. The scattering unit of PHIPS allows for the measurement of the polar light scattering function of cloud particles with an angular resolution of $1^{\circ}$ for forward scattering directions (from $1^{\circ}$ to $10^{\circ}$ ) and $8^{\circ}$ for side and backscattering directions (from $18^{\circ}$ to $170^{\circ}$ ). PHIPS has the advantage of a correlation being established between the different particle habits and their corresponding microphysical properties, which will serve as a reference for other instruments in the future.
The completed setup of PHIPS is presented in this paper together with its operation principle, improved image processing algorithms, and calibration procedures. Preliminary results obtained in two cloud campaigns at the cloud simulation chamber AIDA will be discussed briefly: (1) the HALO02 campaign which was conducted in December 2008 in the temperature range from $-5^{\circ} \mathrm{C}$ to $-70^{\circ} \mathrm{C}$ and (2) the ACI03 campaign which was conducted in October 2009 in the temperature range from $-5^{\circ} \mathrm{C}$ to $-40^{\circ} \mathrm{C}$. In a series of experiments ice crystals were grown in AIDA under distinct temperature and saturation ratio conditions. The temperature-dependent morphology changes and the supersaturation-dependent structural complexity were monitored by PHIPS. Habit classification, structural details, and particle orientation were deduced from the image data and discussed in the context of the corresponding scattering data. The scattering function measurements reveal ice particle orientation-dependent specular reflection peaks which might contain information about the surface roughness (Shcherbakov et al., 2006a). We will concentrate here on the technical description of the probe using some selected results for the proof of concept only. A detailed description of the AIDA instrumentation and the experimental procedure can be found in Möhler et al. (2003).

\section{Setup, operation and detection of PHIPS}

\subsection{Setup}

As mentioned in the previous section, the prototype PHIPS imager has been extended by the second imaging unit and the polar nephelometer. The completed setup is shown schematically in Fig. 1. Individual cloud particles reach the detection volume through a sampling tube of $10 \mathrm{~mm}$ inner diameter. These particles intersect the path of a collimated continuous wave laser beam (CrystaLaser, model CL532-300-L, $\lambda=532 \mathrm{~nm}, \quad p=300 \mathrm{~mW}, w=0.36 \mathrm{~mm}$, divergence $<0.02 \mathrm{mrad}$, linearly polarized) at the scattering center. The scattering center is defined by the center of geometry of the angularly distributed collimating lenses ( $L$ 's) (see Fig. 1). The imaging system consists of two identical imaging devices, each of which includes a zoom objective (Navitar, Model $12 \times$ Zoom w/12 mm F.F. with $2 \times$ magnification tubes) and a digital camera (PCO Imaging, model Pixelfly qe). The digital camera has a scan area of $9.0 \mathrm{~mm} \times 6.6 \mathrm{~mm}$, pixel size of $6.45 \mu \mathrm{m} \times 6.45 \mu \mathrm{m}$, and the number of pixels equals $1392 \mathrm{px} \times 1024 \mathrm{px}$. The zoom objective of each imaging device was adjusted to an overall magnification of $8 \times$. The maximum sampling rate of the imaging system is 10 images for each camera.

The collimated laser beam crosses the scattering center with a beam waist of $0.5 \mathrm{~mm}$. A particle detector (not included in Fig. 1) is placed closely to the scattering center in the plane of scattering with its objective oriented towards 


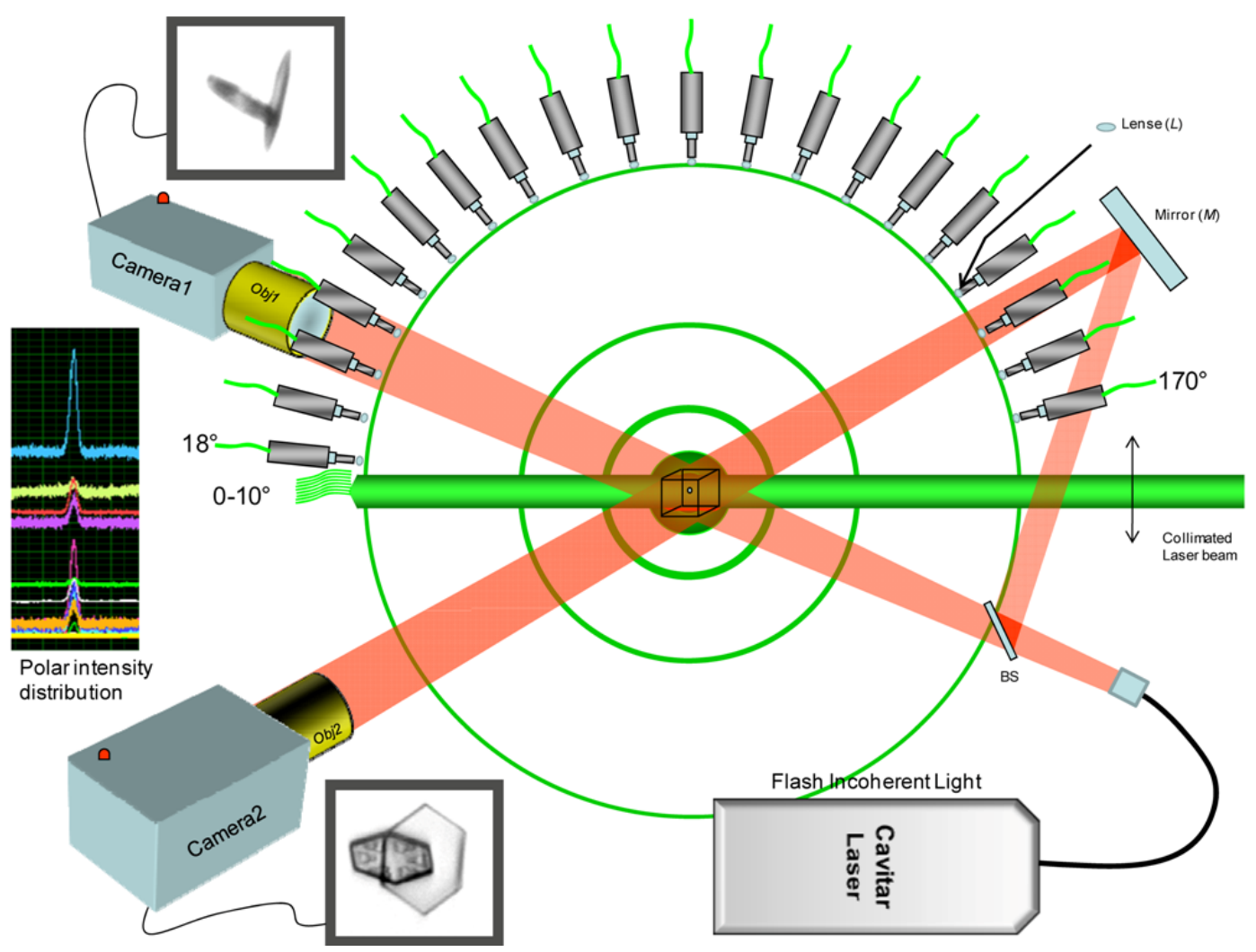

Fig. 1. Optical scheme of PHIPS. The scattering plane includes the incident laser beam (green), polar detectors and particle detector (not drawn) and is perpendicular to the stream of particles allowed to travel through PHIPS. The forward scattering signals are collected using 10 bare plastic fibers placed at a radial distance of $200 \mathrm{~mm}$ from the scattering center with an angular resolution of $1^{\circ}$. An extra bare fiber is placed at $0^{\circ}$ for alignment purposes. The side and backscattering signals are collected using 20 plastic fibers coupled to collimating lenses $(L)$ of focal lengths $=15 \mathrm{~mm}$ placed at a radial distance of $60 \mathrm{~mm}$ from the scattering center, with an angular resolution of $8^{\circ}$. All fibers have a radius of $1 \mathrm{~mm}$. The imaging plane includes the two cameras, the incident laser beam and path of particle stream. Each camera is equipped with an objective which has its focal plane at the scattering center. The Cavitar laser is used as illumination (red) for the imaging system where the beam is divided equally into two paths at the beam splitter (BS).

the scattering center perpendicularly to the laser beam. The particle detector is described in detail in Schön et al. (2011). However, the slit aperture placed at one focal point of the integrated telescope is replaced here by a pinhole (diameter $=200 \mu \mathrm{m})$ to adapt the two-camera configuration. The detection volume, (Fig. 2), is defined by the diameter of the pinhole projection on the imaging plane and the beam waist at the scattering center and is limited by three volumes: $(1,2)$ the depth of field (DOF) in the objective focal plane times the field of view (FOV) for each camera-objective combination and (3) the scattering volume which is defined by the laser beam waist diameter at the scattering center $(0.5 \mathrm{~mm})$ and the diameter of the particle inlet $(10 \mathrm{~mm})$. The FOV corresponding to the camera scan area has a height of $\mathrm{FOV}_{\mathrm{h}}=1.125 \mathrm{~mm}$ and width of $\mathrm{FOV}_{\mathrm{w}} \sim 0.825 \mathrm{~mm}$ at $8 X$ magnification. This value may change slightly depending on the exact magnification of the telescope unit. The DOF of the prototype particle imager of PHIPS was found to be $300 \mu \mathrm{m}$. However, a smart edge detection algorithm has been developed to increase this value to $1000 \mu \mathrm{m}$, as will be shown below (see Sect. 4.1.3). The two identical imaging devices are placed symmetrically around the incident laser beam in the forward direction with $30^{\circ}$ angles from both sides. Such a construction simulates the human ocular system which empowers 3-D vision. To improve the homogeneity of the image background and the quality of the image, the ultrafast illumination flash lamp of the prototype version of PHIPS was replaced by an incoherent pulsed laser source (CAVITAR, $690 \mathrm{~nm}$ ). A long-passfilter (THORLABS, FEL0600) is used to prevent the scattered light from disturbing the captured images. The advantage of using laser illumination instead of a flash lamp is the high pointing and power stabilities from one image to another and the prevention of chromatic aberrations. The incoherency is mandatory to avoid any interference patterns on the images.

The scattering plane is perpendicular to the object plane of the imaging system and defined by the arrangement of polar detectors. The particle flux is perpendicular to the scattering 


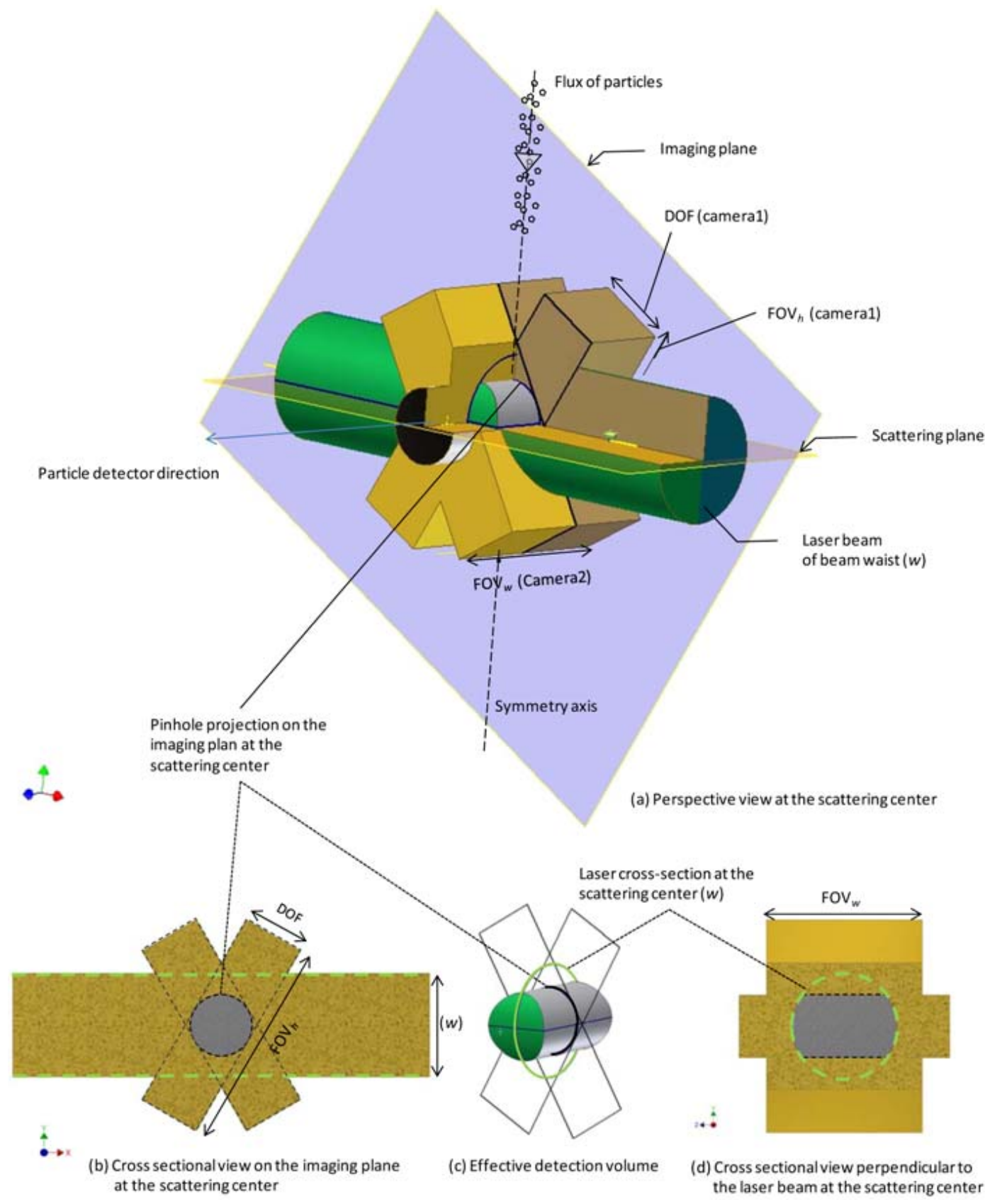

Fig. 2. The intersection of the laser beam, the FOV $\times$ DOF and the projection of the pinhole (placed in the particle detector optics) at the scattering center. (a) A perspective view, (b) a cross sectional view of on the imaging plane at the scattering center, (d) a cross sectional view perpendicular to the laser beam at the scattering center, and (c) the effective detection volume of PHIPS. DOF: Depth-of-field, FOV $\mathrm{h}$ : Field-of-view height, and $\mathrm{FOV}_{\mathrm{w}}$ : Field-of-view width. DOF (camera2), $\mathrm{FOV}_{\mathrm{h}}$ (camera2) and $\mathrm{FOV}_{\mathrm{w}}$ (camera1) are not indicated on the drawing for clarity.

plane. The angular resolution of the differential scattering function is determined by the size and positions of the detectors. It is $1^{\circ}$ for forward scattering directions (from $1^{\circ}$ to $10^{\circ}$ ) and $8^{\circ}$ for side and backscattering directions (from $18^{\circ}$ to $170^{\circ}$ ). The light scattering pulses at the individual scattering angles are transmitted via $1 \mathrm{~mm}$ polymethyl methacrylate
(PMMA) fibers to an optoelectronic amplifier array. Due to the generally high scattering intensity and its strong angular dependence in near-forward scattering direction, a stack of 11 bare PMMA fibers is used to cover the $0^{\circ}$ to $10^{\circ}$ angular range. To enhance the side and backscattering signals, each fiber is equipped with a collimating lens $(L)$ of $60 \mathrm{~mm}$ 
focal length. The solid angle of each channel from $0^{\circ}$ to $10^{\circ}$ is $2 \times 10^{-5} \mathrm{sr}$ and from $18^{\circ}$ to $170^{\circ}$ it is $5.5 \times 10^{-3} \mathrm{sr}$. The $0^{\circ}$ fiber is used for the alignment of the incident collimated laser. The transmitted light is observed by a common photodetector or even visually to maximize the light signal at $0^{\circ}$ using a pinhole placed at $180^{\circ}$ and two counter mirrors that direct the laser beam from the laser source to the detector along the $0-180^{\circ}$ axis. The optoelectronic amplifier consists of three units. Each unit includes ten channels of optoelectronic transducers (GE Intelligent Platforms, OE-200-SI Variable Gain Photoreceiver) equipped with fiber couplers. The 30 channels are connected to a 32-channel high-speed acoustic data acquisition computer card (ICS-645B) which reads the produced electric signal upon each trigger with a maximum sampling rate of 262,144 samples/channel, if all 32 channels are active. The analog-to-digital converters (ADCs) of the card are operated in the capture mode with a pre-trigger data storage sub-mode. Using this mode, the ICS-card stores samples continuously before the trigger and acquires a programmable number of samples following the trigger. This, allows for the recording of the scattering signal produced by the same particle which triggers the system.

\subsection{Principle of operation}

PHIPS uses an automated particle event triggering system ensuring that only those particles are captured, which are located in the field of view - depth of field volume of the microscope unit. Once a particle intersects the collimated laser beam, the particle detector sends a primary trigger signal to the TTL trigger generator. Three TTL trigger signals are generated and sent to the scattering acquisition electronics card (SAEC), the shutters of the two cameras, and the pulsed incoherent illumination laser (PIIL), with different time delays $(\Delta \tau)$ being determined depending on the particle speed. Practically, $\Delta \tau=0$ for SAEC and camera shutters and $\Delta \tau=25 \mu$ s for the PIIL. The $25 \mu$ s is the maximum latency of the CCD after the exposure trigger. The additional latency of the PIIL $(0.5 \mu \mathrm{s})$ guarantees the capture of particle after the start of CCD exposure. The exposure time (shutter open) of each camera is set to $100 \mu$ s, while the pulse width of the flash laser is set to $40 \mathrm{~ns}$ for a particle travelling through the probe with a velocity of $2 \mathrm{~m} \mathrm{~s}^{-1}$. This combination allows for the capturing of the particle within the fieldof-view of the cameras with high spatial resolution. At the particle velocity mentioned, the spatial displacement during imaging is only $80 \mathrm{~nm}$. For faster particles, a shorter pulse width of the PIIL can be used. The image is recorded in gray scale as an $8 \times$ magnified bright field image where the flash source, the particle, and the telescope-camera combination are placed successively on one line when the particle is in the scattering center.

The output of the detection process is a raw data set consisting of the polar scattering intensities data file and two images for each imaged particle. Since the scattering acquisition rate is higher than the imaging acquisition rate, the number of correlated data is limited by the acquisition rate of the imaging part. PHIPS is equipped with a complementary homemade software package to generate the scattering phase function and a set of morphological parameters like area, perimeter, waddle disk diameter, maximum feret diameter... etc. for each image. Moreover, a 3-D model can be constructed for spheres, plates, and columns as will be discussed in detail below (see Sect. 4.1.6). An important advantage of constructing a 3-D model of the ice particle is the possibility of determining an exact size (or particle dimensions) independently of the particle orientation relative to the camera, which has a big influence on the area projected on the CCD chip, if only a single camera is used. Even in the case of more complex habits (e.g. particle aggregates), where construction of a 3-D model from two cameras is impossible, it is still possible to reduce the scatter in results when plotting size distribution, for example, by selecting the larger evaluated size from the two images of each particle. The software package of PHIPS is programmed to optionally select the largest, smallest or averaged value of a parameter extracted from two corresponding images.

\subsection{Detection volume and volume sample rate}

The volume sample rate is defined by the cross sectional area $A_{\mathrm{s}}$ of the detection volume in the scattering plane and the particle speed. As mentioned above, Fig. 2 shows a cross section of the overlapping volumes at the scattering center. In order to select only those particles that are within the depthof-fields of both cameras, a pinhole is used in the particle detector to confine the detection volume. The pinhole diameter $\left(d_{\mathrm{ph}}\right)$ and, hence, area $\left(A_{\mathrm{ph}}=\pi\left(\frac{d_{\mathrm{ph}}}{2}\right)^{2}\right)$ is selected to fit in the intersection area of the two DOF $\times \mathrm{FOV}_{\mathrm{h}}$ planes of both imaging units and not to exceed the laser diameter (see Fig. 2b). The trigger detector uses a telescope of magnification power $=0.5$. This results in an image of the pinhole with a diameter $d_{\mathrm{s}}=d_{\mathrm{ph}} / 2$ (or area $A_{\mathrm{s}}=A_{\mathrm{ph}} / 4$ ) at the scattering center. The diameter of the largest inscribing circle of this intersection area is $0.5 \mathrm{~mm}$ for the current PHIPS design. The intersection between a cylinder of $A_{\mathrm{S}}$ base area and $\mathrm{FOV}_{\mathrm{w}}=0.8 \mathrm{~mm}$ height with a beam of $w=0.5 \mathrm{~mm}$ results in a quasi cylindrical detection volume (see Fig. 2c) of:

$V_{\mathrm{d}} \simeq A_{\mathrm{s}} \times w$

$V_{\mathrm{d}}$ was about $0.004 \mathrm{~mm}^{3}$ (corresponding to a $200 \mu \mathrm{m}$ pinhole diameter) during the two campaigns discussed here and has a maximum of $0.1 \mathrm{~mm}^{3}$ for the current PHIPS design (this can be achieved using a pinhole of $1 \mathrm{~mm}$ diameter). This volume can be increased by decreasing the magnification of the microscope units and increasing the pinhole diameter accordingly, which might be useful for detecting the few large ice crystals in mixed-phase cloud situations. The volume can be increased even further by several orders of magnitudes by increasing the laser beam diameter when using PHIPS as an 
ordinary polar nephelometer, i.e. without scattering-imaging correspondence. However, as mentioned above, this is not the aim of PHIPS, since this instrument is planned to correlate the particle habits to the optical microphysical properties. The volume sample rate is defined as the flux of particles crossing the sensitive area:

Volume sampling rate $=A_{\mathrm{s}} \times v_{\mathrm{p}}$,

where $v_{\mathrm{p}}$ is the velocity of the particles moving through the sensitive area. This article presents exemplary results of AIDA cloud chamber studies for a particle flux of $2 \mathrm{~m} \mathrm{~s}^{-1}$ that results from pumping air through the PHIPS housing (see Schön et al., 2011). In addition, $A_{\mathrm{s}}$ has a maximum value of $2.5 \times 10^{-3} \mathrm{~cm}^{2}$ corresponding to the current PHIPS magnification, beam waist, FOV, and DOF. The resultant maximum volume sampling rate is then $0.5 \mathrm{~cm}^{3} \mathrm{~s}^{-1}$.

\section{Calibration}

\subsection{Imaging system}

The imaging system is calibrated by a well-defined fiber optics placed in the scattering center. The calibration value differs from one experiment to another depending on the exact magnification used by the telescope units (see above). This value was $0.74 \mu \mathrm{m} \mathrm{px}^{-1}$ for the HALO02 campaign and $0.79 \mu \mathrm{mx}^{-1}$ for the ACI03 campaign. Oversizing and undersizing may occur due to a slight out-of-focus position of the particle and is probably dependent on the particle size. This effect is discussed in detail in the image analysis section.

\subsection{The polar scattering system}

The calibration of this unit depends on the quality of the imaging calibration. Water droplets are allowed to pass through the probe and detected by the pre-calibrated imaging system as well as the polar scattering system. Knowing the exact size of the water droplets from the imaging system allows for the evaluation of the scattering phase function using the well-known Mie theory. Comparing the experimentally obtained scattering phase function with the theoretical one gives a series of calibration factors, each of which corresponds to an individual channel of the 30 polar scattering channels. This procedure is easier and more reasonable than those that use theoretical calculations including solid angles and optical apertures of the detectors only, since it also considers the artifacts like the lateral displacement of the collimating optics and the corresponding partial overlaps of the focal points.

Figure 3 shows a comparison between the experimentally obtained scattering intensity of light versus polar angle and the theoretical curve for a water droplet of $94 \mu \mathrm{m}$ diameter. A disadvantage of the used laser is its narrow spectral line width (about $0.15 \mathrm{~nm}$ or less). This narrow spectral line width results in the strong oscillations seen on the theoretical curve

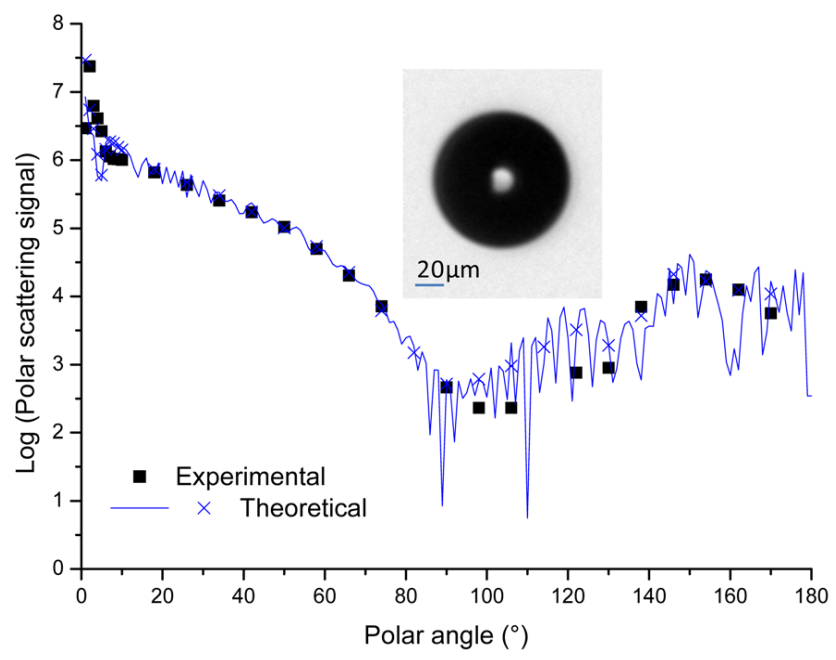

Fig. 3. Comparison between the experimentally obtained scattering intensity of light versus polar angle and the theoretical curve for a water droplet of $94 \mu \mathrm{m}$ diameter. Crosses are the averages of theoretical values over the solid angle covered by the corresponding detector.Also keep in mind that the log scale emphasizes low intensity values and, hence, the difference between experiment and theory in our case.

of the angular scattering intensity of a single particle, Fig. 3 and also Fig. 13, which will be discussed in Sect. 5.2. However, a preliminary study of the lowest acceptable spectral line width, which can reduce these oscillations sufficiently, revealed that a laser of a spectral line width of about $2 \mathrm{~nm}$ (like that used by Gayet et al., 1997) would be good enough along the side scattering angles (from about $30^{\circ}$ to $140^{\circ}$ ). The wider the spectral line width, the smoother are the angular scattering intensities of a single particle. Smoothing over the full range (forward, side and back scattering) would be achieved, if the spectral line width was higher than $5 \mathrm{~nm}$. Obtaining such a laser with such a spectral line width and meeting all other requirements (like compact size, low beam divergence ... etc.) will be one of the challenges of the PHIPSHALO phase.

As the sampling volume has a finite size, the optical system is not stigmatic. Therefore, a particle located outside the focal point in the sampling volume scatters energy following a certain angle which may not reach the corresponding detector. A numerical simulation is made in order to evaluate subsequent errors of the measurements. The effect of the final scattering volume (results from Monte-Carlo GO ray-tracing simulations) on the measured scattering signal is given here: for the $1-10^{\circ}$ detectors, $24.16 \%$ of the bin intensity are lost due to the final dimension of the scattering volume (rays of the corresponding scattering angles do not reach the detector). However, the same amount is gained from outside. The maximum acceptance half angle is effectively increased from $0.143^{\circ}$ to $0.242^{\circ}$ (however, only $12.86 \%$ of the rays in this extra angular region hit the detector). For the $18-170^{\circ}$ 
detectors, $4.87 \%$ of the bin intensity are lost due to the final dimension of the scattering volume (rays of the corresponding scattering angles do not reach the detector). However, the same amount is gained from outside. The maximum acceptance half angle is effectively increased from $2.386^{\circ}$ to $2.715^{\circ}$ (however, only $16.52 \%$ of the rays in this extra angular region hit the detector).

\section{Data analysis}

\subsection{Image analysis and 3-D morphology}

The data acquisition software triggers the system at each predefined period of time to record background measurements (blank images at the CCDs and background light at the angular scattering sensors) during each experiment. The background images are averaged and subtracted from the raw images. As a result and thanks to the homogeneity and pointing stability given by the PIIL, a couple of sharp and splotchesfree images can be obtained for each particle. On this basis, the region of interest (ROI) which includes the particle can be located. Each image is then converted into a binary image where pixel values are placed as either 0 (black) for background light or 1 (white) for particle. The image analysis process uses the steps and methods of the specially developed algorithm described by Schön et al. (2011), except for those described below.

\subsubsection{Extracting area(s) of interest from each image}

In contrast to the algorithm referred to above, our algorithm does not only process the object generating the steepest gradient, but also those of lower but still sufficient steepness, if more than one particle is imaged by one shot. It is rare that more than one particle are detected within one image, as the probability of two particles being accidentally present in the tiny volume of detection at the same time is low. This is unfavorable for the correlation process with the polar scattering signals. Nevertheless, these particles are not ignored, but extracted and stored in different files and registered to the same time of detection. This supports the size distribution studies.

\subsubsection{Evaluation parameters}

In addition to the area, aspect ratio, roundness, and equivalent disk diameter, an additional algorithm was conceived to evaluate the exact length, orientation, and basal-facetdiameter of columns. This algorithm applies a stepwise rotation to the image, measures the length and width of the particle-bounding-rectangle (PBR), and locates the rotation angle where the maximum length/width ratio of the PBR is found. This angle defines the orientation of the columnar particle relative to the original orientation of the image and its length and basal-facet-diameter equaling the length and width of the PBR, respectively. The PBR is defined as a rectangular region, the sides of which are parallel to the coordinate axes and bounds certain object. The function of defining the PBR is used as it is included in the LabView environment and in particular in the IMAQ package which is used to perform the calculations described here. Determination of column-like particle dimensions and orientation is required for the stereo imaging and 3-D morphology which is discussed in a later section.

\subsubsection{Edge detection and out-of-focus particles}

Two different histogram-based edge detection methods were used by Schön et al. (2011) to detect the particle edge: (1) the triangle algorithm applied to the gradient image to effectively find the ROI and (2) the isodata algorithm applied to the ROI image in order to do the final particle segmentation. The latter is an iterative method to find the gray scale threshold value, but it has problems with small and out-of-focus particles. In the case of small particles, their images are faint and produce a flat broad distribution. In the case of out-of-focus particles, the histogram method fails, since the peak in the histogram corresponding to the particle is masked by the histogram background. So, there is an urgent need for a method that can correct for small and out-of-focus particles or at least distinguish between in- and out-of-focus particles. It is also necessary to define the acceptable displacement from the focal plane, which has no significant influence on the particle size evaluation.

In order to find a better edge detection algorithm, we started to investigate how human beings define the particle edge in sharp and out-of-focus images. From these investigations, we can conclude that humans automatically search (or detect) a point where the trend of the gray scale (in the image) changes curvature. The only point which satisfies this criterion is the inflection point (IP) of the gray scale slopes at the edge of the particle. The inflection point is a unique point on the rising gray scale at the edge of a particle and corresponds to the peak point on the curve of the first derivative of the gray scale value with respect to the distance along a line perpendicular to the edge at any point. The slope at the inflection point reflects the sharpness of the image. The sharper the image, the steeper is the slope of the gray scale gradient at the particle edge, Fig. 4.

To test how the evaluation of the particle size is influenced by its displacement from the focal plane under this new edge detection concept, an auto scanning-and-imaging system was developed. A piezo-driven nozzle which produces water droplets of constant size and predefined rate is used as a source of identical particles. The piezo-driven nozzle was attached to a motorized translation stage (THORLABS, Z725B motorized DC servo) moving along the optical axis of the imaging system. A simple program was written using LabView to move the piezo with predefined steps $(10 \mu \mathrm{m} / \mathrm{step}$ for the results presented) along the optical axis and accumulate a number (about 10 for the results presented) of images/step. 

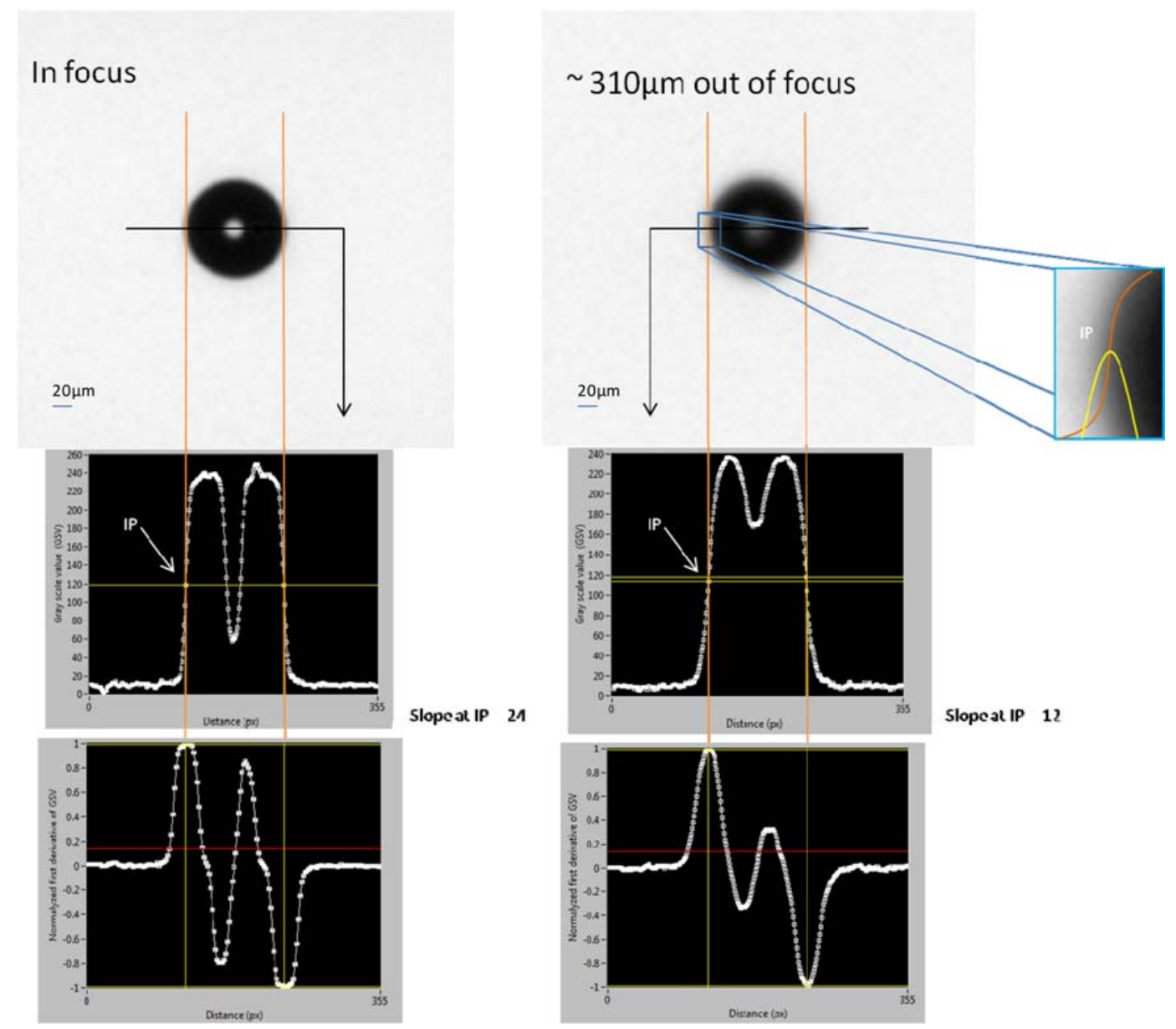

Fig. 4. Gray scale variation at the edge of a spherical particle (a $94 \mu \mathrm{m}$ water droplet) placed in focus (left panel) and $310 \mu \mathrm{m}$ out of focus (rightpanel) positions within the field of view of the PHIPS camera objective. The two upper graphs represent the gray scale values versus distance. The two lower graphs represent the normalized partial derivatives of gray scale values versus distance in pixel unit on the CCD chip. Distances are measured in pixel with respect to an arbitrary point positioned on the left side of the particle.

The particle sizes were calculated using the algorithm discussed above. The slope of the gray scale variation at the IP is also calculated at each step. (The presented values are the averages of those obtained at each step). It is worth mentioning that the piezo-driven nozzle produced identical droplets with diameter values fluctuating around $94 \mu \mathrm{m}$ at a standard deviation of $\mathrm{SD}=0.66 \mu \mathrm{m}$ only.

Figure 5 shows the dependency of the investigated particle diameter on the spatial position of the particle with respect to the focal point. It also shows the slope of the gray scale gradient at the IP, which can be fitted by a Gaussian profile. The variance in the droplet diameter investigated using the inflection point algorithm from images collected at a magnification $M=8 \times$ is found to be $4 \%$ of the particle size over a range of $1000 \mu \mathrm{m}$ around the focal point. This means that the DOF is about $1000 \mu \mathrm{m}$, which is three times larger than that obtained by Schön et al. (2011). The vertical lines at $d_{-}$and $d_{+}$show the former limits of the DOF of PHIPS. It is worth mentioning that improving the background (image quality in general) using the incoherent laser contributed to this enhancement. The advantage of a Gaussian fit trend is that a threshold of the slope at the IP can be defined, above which the particle is considered and below which the particle is omitted. In contrast to Schön et al. (2011), the two different histogram-based edge detection methods were replaced by one IP-based method applied to the gradient image to effectively find the region of interest (ROI) and to do the final particle segmentation simultaneously.

\subsubsection{Particle size dependence}

In order to analyze the influence of the particle size on the validity of the size evaluation procedure, the developed algorithm was applied to the same set of images which were selected by Schön et al. (2011). In this way, increasing overestimation with decreasing particle size was studied. These are standard well-defined tiny (several microns) ice analogues (Ulanowski et al., 2003) made of sodium hexafluorosilicate 


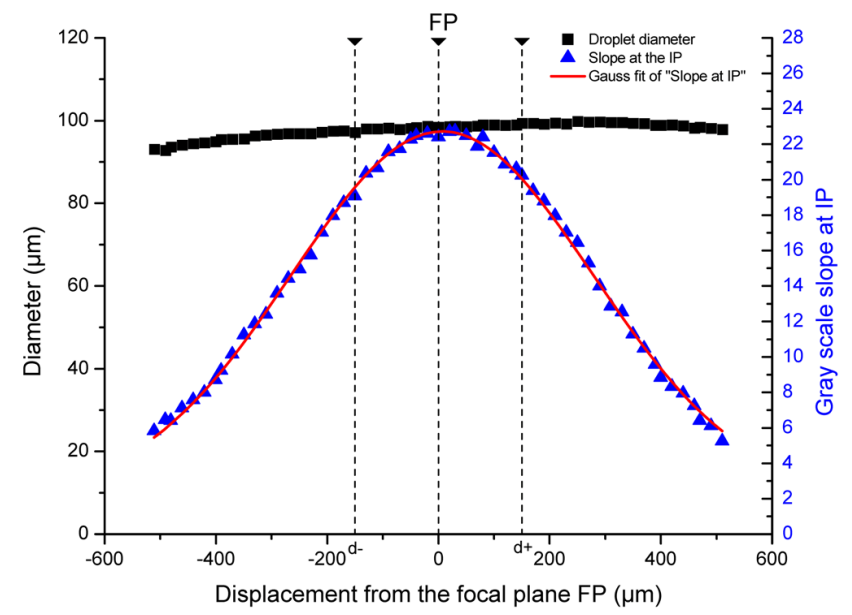

Fig. 5. The calculated particle diameter (of a water droplet) versus the displacement from the focal plane of the imaging system used in PHIPS (black solid squares), the slope of the gray scale variation at inflection point around the particle edge (blue solid triangles), and its Gaussian fit (red line). The vertical dashed lines at $\mathrm{d}-$ and $\mathrm{d}+$ show the former limits given by Schön et al. (2011) of the DOF obtained before using the pulsed incoherent illumination laser and the inflection point edge detection algorithm.

(Na2SiF6). These crystals exhibit the shape of hexagonal columns and plates as well as complex combinations of these two basic structures like those observed for ice crystals. In addition, their refractive index is similar to that of ice $(\sim 1.31)$. However, $\mathrm{Na} 2 \mathrm{SiF} 6$ is completely stable at room temperature, which is not the case for ice. As shown in Fig. 6, the investigated particle sizes agree well with those given as a standard values with a $\mathrm{SD}=3 \%$ of the investigated/given size ratio along a size range from 9.4 to $187.8 \mu \mathrm{m}$. It is worth mentioning here that these images were collected using the old lighting system (flash lamp), which is more or less the origin of the small fluctuations around the mean value.

\subsubsection{Particle classification}

It is necessary to distinguish particles according to their habits in order to estimate the contribution of a certain habit to a meteorological phenomenon. A homemade computer program has been developed using LabView to classify particles according to their habit. For instance, the function of selecting columnar particles out of a set of images was tested as shown below in experiment HALO02_18. The program is based on an algorithm that works on the set of binarized images. In contrast to other algorithms which use a single parameter only (e.g. circularity factor and aspect ratio) as a classification criterion, the developed algorithm uses a mix of criteria, each of which contributes with a certain weight to the final decision of whether a particle is columnar or not. Figure 7 shows a flowchart of the evolution of the decision on the particle habit. The particle dimensions undergo three

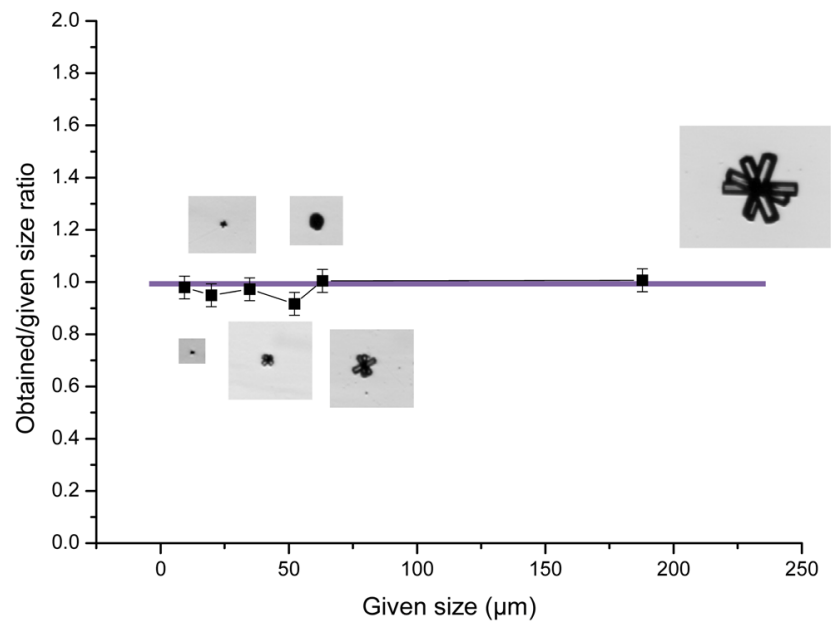

Fig. 6. The ratio of the evaluated particle sizes (using the developed inflection point algorithm) to those given as standard values for standard ice analogues.

tests: (1) particle area $(A)$ to bounding rectangle area (BRA) ratio $\left(R_{\mathrm{A}}\right),(2)$ aspect ratio $(\mathrm{AR})$, and (3) the Heywood circularity factor $\left(F_{\mathrm{c}}\right)$. The particle should have an area close to its BRA (test 1) or a small aspect ratio (test 2) and not be circular (test 3 ) to be a columnar particle with high probability. If test 3 shows that the particle is a circle, the process stops and the particle is classified to be non-columnar. Otherwise, the process proceeds towards the weight box. In the weight box, the weights of the tested criteria $\left(W_{\text {Test1 }}, W_{\text {Test2 }}\right.$, and $\left.W_{\text {Test3 }}\right)$ are measured. The sum of weights should exceed a certain threshold value $\left(W_{\text {th }}\right)$ to draw the final conclusion that the particle certainly is a column; otherwise, it is defined as a non-column. All threshold values are selected carefully to meet the requirements of sorting columns out from a set of images. These values may differ from one experiment to another depending on the imaging quality. However, suitable values used to sort out columns in the HALO02_18 experiment are $R_{\mathrm{ATh}}=0.7, \mathrm{AR}_{\mathrm{Th}}=0.4, F_{\mathrm{cTh}}=1.04$, and $W_{\text {th }}=0.12$. The result was controlled manually by browsing the classified columnar and non-columnar particles visually and it was found that the classification had been free of errors.

\subsubsection{Stereo imaging and 3-D morphology}

During a series of AIDA campaigns, the problem of habit confusion turned out to be caused by particle orientation (e.g. a plate can be seen as a column when its plane is located perpendicular to the FOV of a single camera). This uncertainty was reduced by stereo imaging in PHIPS. In the case of pristine ice crystals like columns and plates, the 3-D habit and particle orientation relative to the scattering plane of the nephelometer could be reconstructed using the information extracted from the two corresponding images of the stereo imaging system. A direct advantage of obtaining two images 


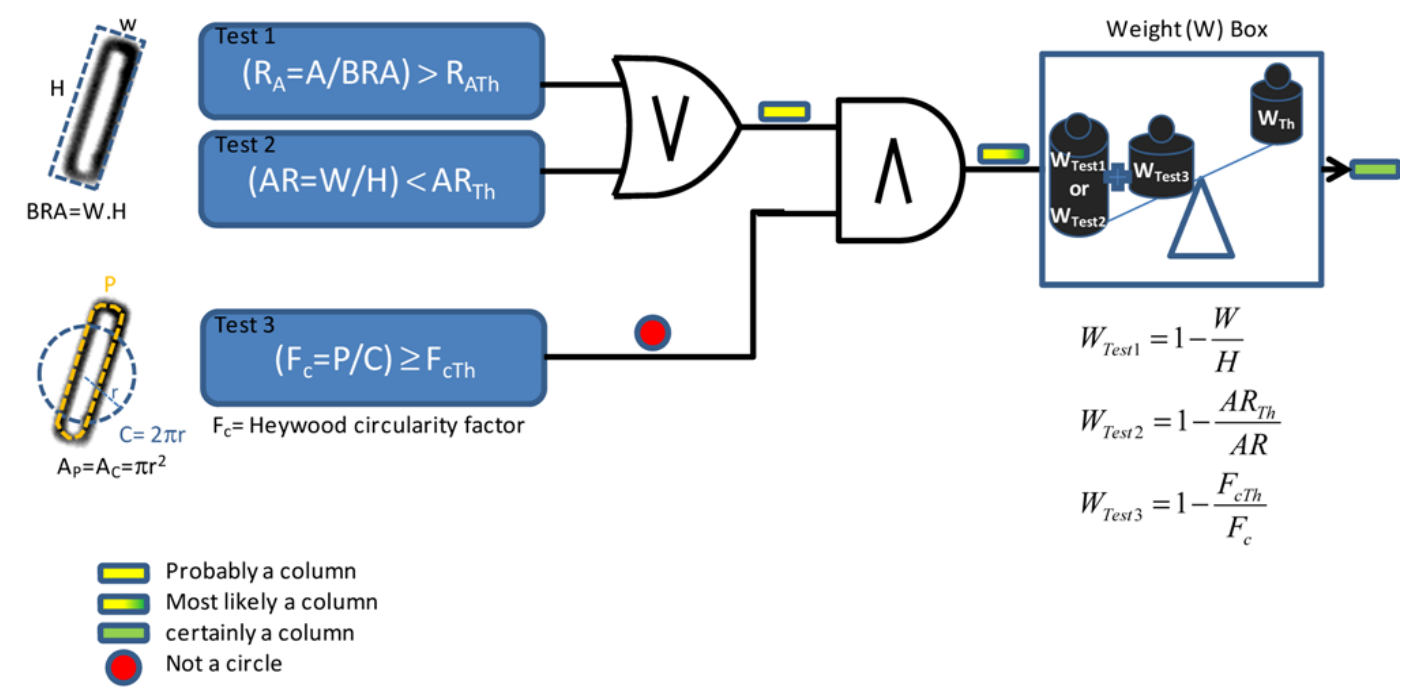

Fig. 7. Particle classification. A flowchart illustrates the algorithm used to sort out columnar particles from a set of particle images. Three tests contribute to the final decision with different weights; test 1: the ratio $\left(R_{\mathrm{A}}\right)$ between the particle area $(A)$ the bounding rectangle area (BRA), test 2: the aspect ratio (AR) and test 3: the Heywood circularity factor $\left(F_{\mathrm{c}}\right)$. W: denotes the weight of the test. It is a measure of how far the tested criterion is satisfied. The Heywood circularity factor is the ratio between particle perimeter and the circumference of equal area circle. The subscript (th) denotes the threshold value of the corresponding parameter.

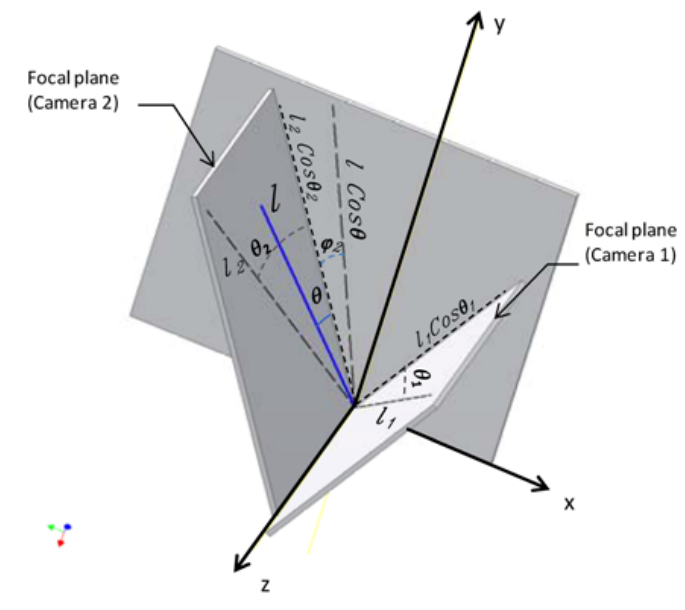

(a)

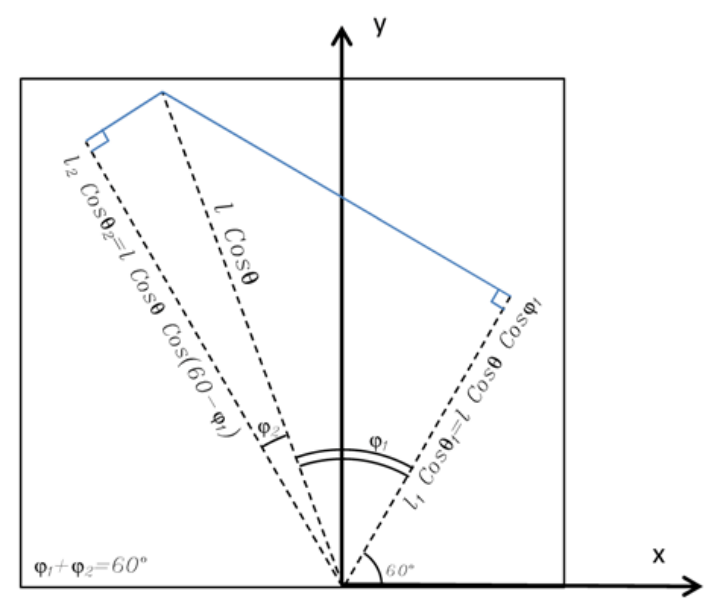

(b)

Fig. 8. The projections of a column of length $l$ on two focal planes: (a) perspective view, (b) normal view along the common axis of intersection $(z)$. The laser beam propagates along the $\mathrm{x}$-axis. The particle stream travels along the y-axis. The angle between the two focal planes is $120^{\circ}$ from the laser side $60^{\circ}$ from the particle stream side.

for the same particle at two different angles is that the investigated parameters can be compared and/or averaged. For example, the larger value of particle size from the two corresponding images of plates, columns or complex irregular particles (e.g. rosettes, stars ...) was selected in order to reduce the scatter of points on plots like the size distribution. Averaging can be used in case of spherical shapes, e.g. water droplets, to enhance the quality of results. Furthermore, the most important advantage of the two corresponding images is the sense of 3-D morphology similar to human stereo vision. Constructing a 3-D image of regular geometric shapes like rods, columns, plates... etc. is possible. Figure 8 shows the projection of a simple 3-D column on two focal planes. The inverse process is the construction of a 3-D column from two projections on two focal planes using the following scheme: 
Definitions of known and unknown parameters referring to Fig. 8:

- $l$ : the length of the column, (unknown).

- $l_{1}$ and $l_{2}$ : the lengths of the projections of the column on the focal planes of camera 1 and camera 2, respectively, (known).

- $\theta$ : the angle between the column and the $\mathrm{x}-\mathrm{y}$ plane, (unknown).

- $\theta_{1}$ and $\theta_{2}$ : the angles between the column projections and the $\mathrm{x}-\mathrm{y}$ plane, (known).

- The projections of $l, l_{1}$ and $l_{2}$ on the x-y plane are shown on Fig. 8b.

- $\varphi_{1}$ and $\varphi_{2}$ : the angles between plane of the column and $\mathrm{z}$-axis and the focal planes of camera 1 and camera 2, respectively. This plane is not shown in Fig. 8 for reasons of clarity.

The $\mathrm{z}$-axis is a common axis where the three planes (two focal planes and a plane containing the column and z-axis) intersect. Since camera 1 and camera 2 are placed in one plane perpendicular to the $\mathrm{z}$-axis, the two projections of $l_{1}$ and $l_{2}$ on the z-axis should be equal and equal to the projection of $l$ on the same axis, i.e.:

$l \sin \theta=l_{1} \sin \theta_{1}=l_{2} \sin \theta_{2}$.

From the geometry in Fig. 8a and b, the following relations can be derived:

$\varphi_{2}=60-\varphi_{1}$

$l_{1} \cos \theta_{1}=l \cos \theta \cos \varphi_{1}$

$l_{2} \cos \theta_{2}=l \cos \theta \cos \left(60-\varphi_{1}\right)$.

These are four mathematical relations in four unknowns $(l$, $\theta, \varphi_{1}$ and $\left.\varphi_{2}\right)$, which is why a unique solution is possible. Solving Eqs. (5) and (6) together gives:

$\varphi_{1}=\tan ^{-1}\left(\frac{\frac{l_{2} \cos \theta_{2}}{l_{1} \cos \theta_{1}}-\cos 60}{\sin 60}\right)$.

Using Eq. (4),

$\varphi_{2}=60-\tan ^{-1}\left(\frac{\frac{l_{2} \cos \theta_{2}}{l_{1} \cos \theta_{1}}-\cos 60}{\sin 60}\right)$.

Using the equality (Eq. 3) with Eqs. (5) or (6) gives

$\theta=\tan ^{-1}\left[\tan \theta_{1} \cos \varphi_{1}\right]$

or

$\theta=\tan ^{-1}\left[\tan \theta_{2} \cos \varphi_{2}\right]$.
Finally, $l$ can be obtained from Eqs. (3) or (4) as:

$l\left(l_{1}, \theta_{1}, \theta, \varphi_{1}\right)=\frac{l_{1} \cos \theta_{1}}{\cos \theta \cos \varphi_{1}}$

or

$l\left(l_{2}, \theta_{2}, \theta, \varphi_{2}\right)=\frac{l_{2} \cos \theta_{2}}{\cos \theta \cos \varphi_{2}}$.

In this way, the length and orientation of the column with respect to the two focal planes of the cameras can be determined. However, to determine the orientation of the particle relative to the normal coordinates, $(x, y$ and $z)$, the Euler angles can be used as follows:

Starting from the particle coordinate system $\left(\mathrm{x}^{\prime}, \mathrm{y}^{\prime}, \mathrm{z}^{\prime}\right)$ coincidence with the laboratory system $(\mathrm{x}, \mathrm{y}, \mathrm{z})$ where $\mathrm{x}^{\prime} / / \mathrm{x}$, $\mathrm{y}^{\prime} / / \mathrm{y}$ and $\mathrm{z}^{\prime} / / \mathrm{z}$ and having the column normal parallel to the $\mathrm{X}$-axis, we define the following operations:

1. A clockwise rotation by an angle when looking into the positive direction of the $\mathrm{z}^{\prime}$-axis.

2. A subsequent anticlockwise rotation around the new $\mathbf{x}^{\prime}-$ axis with an angle $=\theta$.

3. Since the column is symmetric around its axis and starts from the coordinate origin, the third rotation $(\gamma$ around the new $y^{\prime}$-axis has no effect on the particle orientation.

4. The diameter of the column can be obtained from one image as the thickness of the shadow line.

In case of plates, the particle shadow is divided into different columns (or lines), each of which is treated as described above. The recombination of these lines in the normal coordinates gives the skeleton of the particle morphology. For example, a hexagonal shape can be represented as three diagonals (three lines) where the length and the orientation of each can be obtained by the described method. Once a 3-D model is constructed, the exact dimensions and orientation of the particle can be defined. This information can be used to interpret the scattering data taken quasi-simultaneously with the images.

\subsection{Scattering phase function}

The time-resolved scattering signal (band) obtained from each channel upon each trigger can be either integrated or peak-selected. Integration enhances the detection at low signals, but its resultant exact value depends on the particle speed. On the other hand, peak detection gives particle speed-independent values, but the noise-to-signal ratio is high, mainly for small particles. The presented results are calculated from band integration and the resulting intensities from the 30 channels are plotted as a function of the scattering angles to be compared to the scattering phase function modeled from the particle habit. 


\section{Representative results}

The full operation of PHIPS was tested at the AIDA facility during two ice cloud characterization campaigns, HALO-02 and $\mathrm{ACI} 03$. We present some of the results demonstrating the capability of PHIPS to probe the different habits of ice crystals., including comparisons to other instruments which participated in these campaigns. In addition, a 3-D model of a hexagonal ice crystal, obtained during the HALO02 campaign, will be represented including a comparison of its measured scattering phase function with modeling results using the Ray Tracing with Diffraction on Facets (RTDF) program (Clarke et al., 2006; Hesse, 2008). It should be mentioned that the images collected in HALO02 are of lower quality than those collected in ACI03 due to the different illumination system. In HALO02, particles were illuminated using the same flash lamp used in the prototype particle imager of PHIPS, while in ACI03, the flash lamp was replaced by the PIIL for the reasons mentioned in Sect. 2.1.

\subsection{HALO02_18 experiment}

This experiment aimed at improving our knowledge of the relation between ice crystal habits and their ability to depolarize light. Specifically, we were looking at the backscattering linear depolarization ratio inferred from in situ light scattering measurements at AIDA with the SIMONE instrument and its correlation with the microphysical properties of the ice crystals measured by two complementary imaging systems, namely, PHIPS of KIT and HOLIMO of ETH Zurich (Amsler et al., 2009).

The HALO02_18 experiment was conducted similarly to the second experiment of the IN11 campaign in December 2007 described in Amsler et al. (2009). In contrast to the IN11_2 experiment where plate-like ice crystals had been investigated, columnar ice particles were analyzed in HALO02_18. These columnar ice particles grew in the AIDA chamber at a temperature between $-5^{\circ} \mathrm{C}$ and $-6.5^{\circ} \mathrm{C}$, which is well within the column regime of the Furakawa morphology diagram for in-cloud ice crystal growth (Libbrecht, 2005). It was intended to have an extended period where the ice crystals could grow and mix with new ice crystal seeds at ice saturation ratios slightly above ice saturation, followed by an extended period where the remaining crystals (after the crystal seed addition had been stopped) could grow under highly supersaturated conditions. In this way, a broad range of aspect ratios $\chi=W / H$ could be investigated in HALO02_18. It is important for the interpretation of the data that the total water concentration, i.e. interstitial water vapor plus condensed water of the cloud particles, is measured at the uppermost level of the AIDA chamber next to the heated spray nozzle that is used to inject supercooled droplets to the chamber volume. Interstitial water vapor measurements by the TDL instrument and cloud particle sampling by HOLIMO and PHIPS are located at the second and the bottom levels, i.e. about $2 \mathrm{~m}$ and $4 \mathrm{~m}$ below the uppermost level, respectively. Consequently, only relatively long periods of cloud droplet injection could be detected by PHIPS and HOLIMO, since the droplets start to evaporate already close to the nozzle.

Figure 9 depicts $p, T, s_{i}$ (the ice saturation ratio), WELAS, HOLIMO and PHIPS size distributions, scattering intensities, and depolarization ratio data on the panels a-f, respectively. Temperature and ice saturation ratio values from AIDA are given with an accuracy of $\pm 0.3 \mathrm{~K}$ and $\pm 5 \%$, respectively. Panel a of Fig. 9 shows the evolution of the mean gas temperature inside AIDA during the expansion cooling experiment. The wall temperature of the vessel stayed rather constant throughout the whole experiment. Ice seed injection took place shortly after $0 \mathrm{~s}$ experiment time for about $600 \mathrm{~s}$ (indicated by the two dashed black vertical lines in Fig. 9), followed by a threefold water droplet injection into the AIDA volume started at the experiment times 960, 1120 and $1320 \mathrm{~s}$ for different durations (dashed blue vertical lines in Fig. 9). The droplet injections lead to a moderate increase of the mean gas temperature by latent head release only for the longest injection period starting at $1320 \mathrm{~s}$ experiment time. Panel $b$ shows the ice saturation ratios versus the interstitial and total water contents inside AIDA.

The injection periods of supercooled water droplets are clearly visible by an increase of the total water content. Meanwhile, the emerging ice cloud reduced the interstitial water vapor content and confined the saturation ratio to icesaturated conditions after a short period of enhanced fluctuations during droplet injection. The interstitial phase remained close to a saturation ratio of 1 throughout the experiment, but exceeded this value by about $2 \%$ after the third and longest water droplet injection period. Panel $\mathrm{c}$ is obtained from the WELAS optical particle counter and shows the abundance of the particles and their optical particle diameters based on droplet calibration measurements. The equivalent particle diameter deduced from HOLIMO and PHIPS images as well as the columns' aspect ratios calculated from PHIPS images are shown on panel d. Note that PHIPS data are presented in two point styles corresponding to columnar and non-columnar ice crystals, which demonstrates the ability of the PHIPS imaging and analysis system to classify particles according to their habits. The gaps between 500 and $600 \mathrm{~s}$ and 1200 and $1500 \mathrm{~s}$ in the PHIPS data are due to computer crashes. In addition, the PHIPS results show that the abundance of columns was dominant all the time and especially after the water droplet injections. The high fraction of columnar ice particles was confirmed by accompanying measurements with the novel cloud probe SID-3 (Kaye et al., 2008) shown in Table 1.

Panels e and $\mathrm{f}$ show the scattered intensity (forward and backward) and the linear depolarization ratios $\delta_{/ /}$and $\delta_{\perp}$ as given from SIMONE. The linear depolarization ratio for parallel incident laser polarization $\delta_{/ /}$reveals low values around 0.14 for the emerging ice cloud at the beginning of 

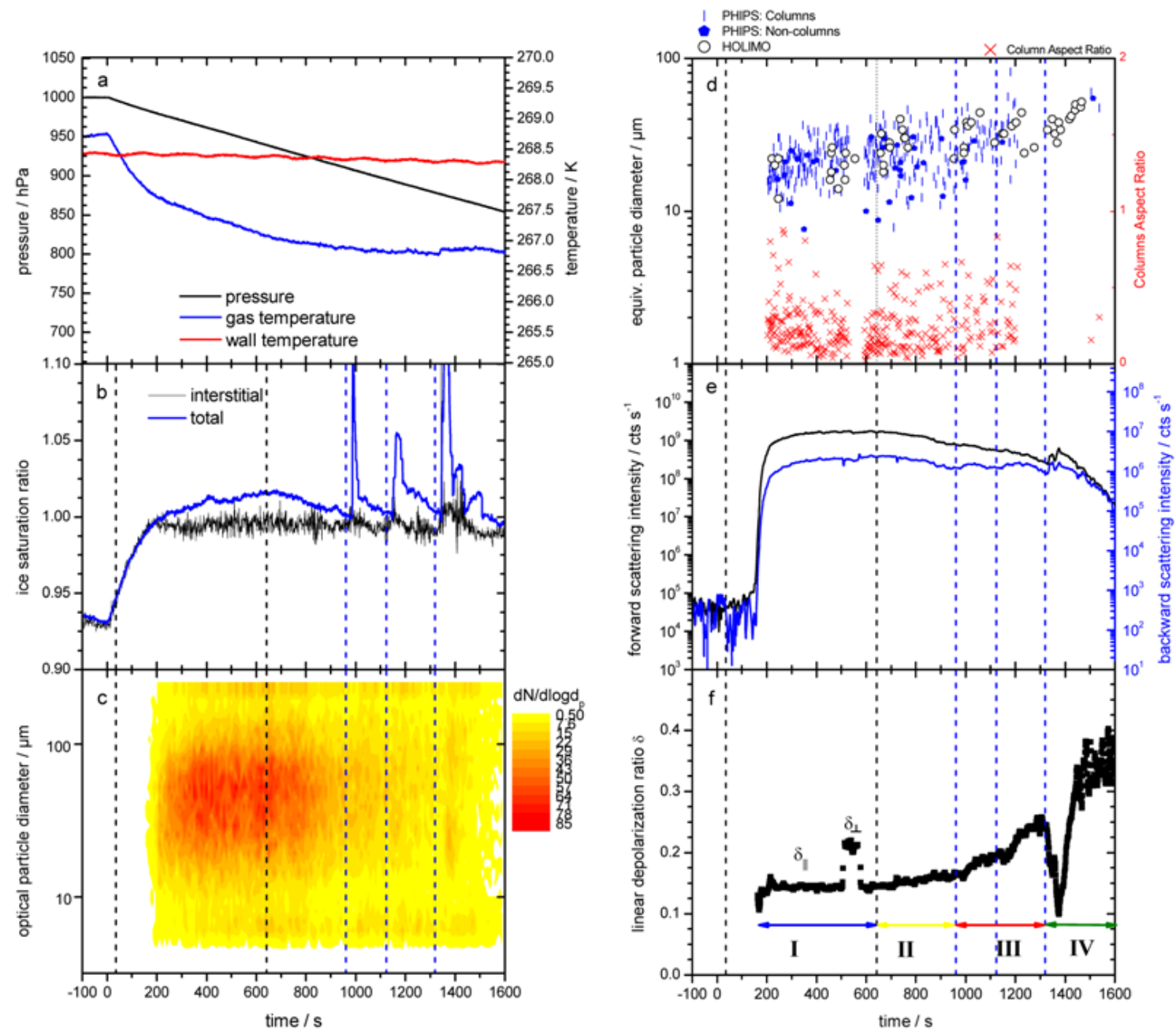

Fig. 9. Temporal evolution of the AIDA mixed phase cloud experiment HALO02_18. Panel (a): wall and gas temperatures and pressure of the AIDA chamber. Panel (b): ice saturation ratio of the total and interstitial water contents si in the AIDA volume. Panel (c): optical particle diameter as measured by WELAS. Panel (d): geometric particle diameters as measured by the HOLIMO and PHIPS imagers together with the aspect ratio solely deduced for the PHIPS detected columns. Panel (e): near-forward $\left(2^{\circ}\right)$ and near-backward $\left(178^{\circ}\right)$ light scattering intensities measured by SIMONE. Panel (f): backscattering linear depolarization ratio measured by SIMONE. See text for details.

Table 1. Fraction of columnar ice particles as given by PHIPS and SID-3 in experiment HALO02_18.

\begin{tabular}{lcc}
\hline & SID3 & PHIPS \\
\hline I & 0.74 & 0.90 \\
II & 0.80 & 0.87 \\
III & 0.78 & 0.92 \\
IV & 0.81 & NA \\
\hline
\end{tabular}

the experiment. Those values increase towards 0.35 at the end of the experiment along with an increasing trend of the mean equivalent ice particle diameter. The linear depolarization ratio shows a decrease every time after supercooled water droplet injection, which is especially pronounced after the third extended period of droplet injection. During this event, the linear depolarization $\delta$ drops down to about the initial value of 0.14 . This means that the period of droplet injection was long enough for the droplets to reach the sensitive volume of SIMONE before they evaporated completely. Hence, SIMONE probed a mixed-phase cloud for a short time. Between 500 and $580 \mathrm{~s}$, the incident laser polarization was changed to be directed perpendicular to the scattering plane. The corresponding linear depolarization ratio $\delta_{\perp}$ is offset by about 0.08 with respect to $\delta / /$.

The ice crystal seed phase was rather long, which is why columnar ice crystals of different sizes were to be expected. This mixture of older columns that may have grown a little during their residence time inside AIDA with younger and smaller ice crystal seeds can be observed on the composite 


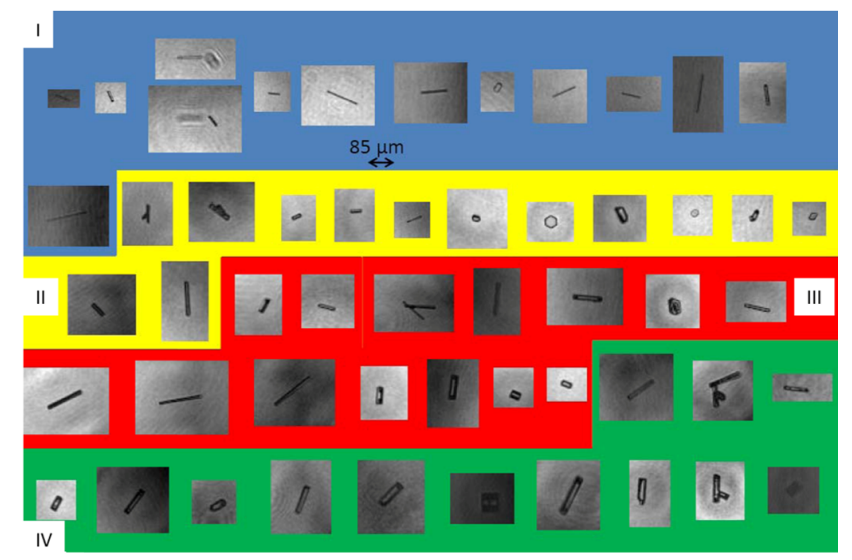

Fig. 10. Ice crystal habits of experiment HALO02_18 obtained by HOLIMO during four different time slots showing phases of habits and frequency of occurrences throughout the experiment.

images of HOLIMO and PHIPS. Sequences of smaller and larger columns are shown chronologically and exemplarily in Figs. 10 and 11 and can be observed especially during the blue period. It is important to emphasize for the following discussion of the measured backscattering linear depolarization ratio that the columnar ice particles observed in blue period are extremely thin with a width in the range of the resolution limit of both imaging instruments. Figure 10 shows a representation of the randomly oriented columnar ice crystals observed by HOLIMO. This random orientation is due to the relatively high velocity $\left(26.5 \mathrm{~m} \mathrm{~s}^{-1}\right)$ with which the sample air is drawn through holographic microscope compared to the moderate velocity of $2 \mathrm{~m} \mathrm{~s}^{-1}$ applied in PHIPS. Such high flow velocities result in a turbulent air profile inside the inlet tube of HOLIMO which also has $10 \mathrm{~mm}$ inner diameter.

During the injection period of the ice seeds, the ice crystals experienced only a slow growth (if any) due to the nearly icesaturated conditions in this time period (see Fig. 9b). This situation was turned into phases of fast growth by the repeated injection of supercooled water droplets. Hence, ice crystal growth via the Bergeron-Findeisen mechanism took place at the nearly water-saturated conditions which prevailed in the vicinity of the spray nozzle at least.

We found a relatively low linear depolarization ratio of $\delta_{\|}=0.14$ for the thin columns observed in the blue and yellow experiment periods of Figs. 10 and 11. Subsequent periods with thicker and larger columns are nicely correlated with the increase observed in the depolarization ratio up to a value of 0.35. A wide range of aspect ratios between about 0.02 and 1 was deduced from the HOLIMO and PHIPS images throughout the experiment (see Fig. 9d). Figure 12 shows theoretical $\delta_{\perp}$ and $\delta_{\|}$values for randomly oriented particles calculated for a scattering angle of $178^{\circ}$ and for an aspect ratio range of 0.0002 (needles) $<\chi<200$ (thin plates). We used the same geometric optics ray-tracing program by Macke et al. (1996) that was applied in the previous

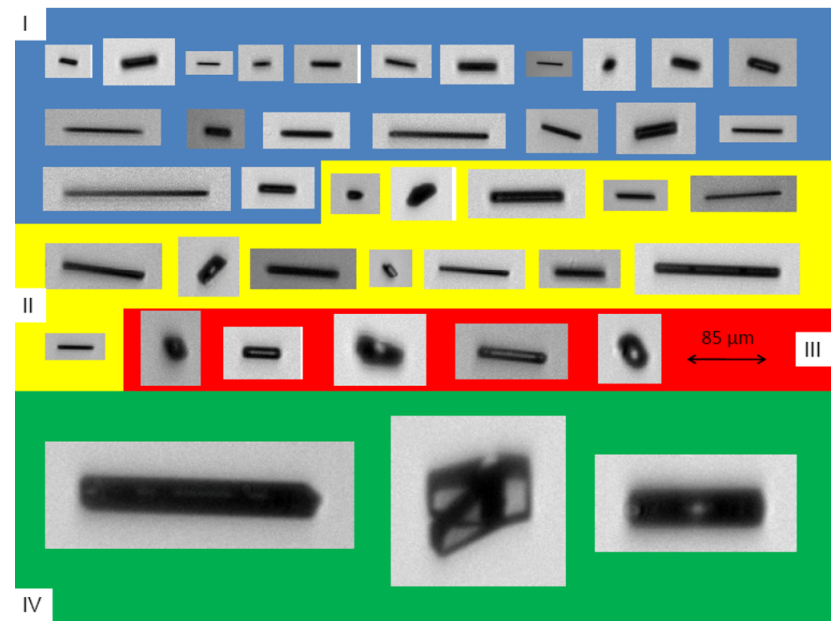

Fig. 11. Ice crystal habits of experiment HALO02_18 obtained by PHIPS during four different time slots showing phases of habits and frequency of occurrences throughout the experiment.

study for plate-like ice crystals (Amsler et al., 2009). For the observed aspect ratio range, the geometric optics model gives a flat $\chi$-dependence of the depolarization ratio $\delta_{\|}$, and $\delta_{\perp}$ values that are always larger than $\delta_{\|}$. These findings are in qualitative agreement with the flat temporal evolution of $\delta_{\|}$and a larger $\delta_{\perp}$ value measured in the blue and yellow periods where the sizes of the ice particles are comparable. Still, the modeled linear depolarization ratios are generally higher than the measured ones. This discrepancy might be partly due to the small width of the investigated columnar ice crystals, which brings geometric optics to the margin of its applicability. A similar observation, i.e. a low depolarization ratio for thin particles, was made for the plate-like ice crystals investigated in the IN11 2 experiment by Amsler et al. (2009). From these results, we can conclude that the observed low depolarization ratios of thin plates and thin columns are rather a consequence of the extreme shape of the hydrometeors than being caused by their actual hexagonal geometry, since we probed two extremes of internal prismatic reflections with hardly any influence of basal or prism facets in either case.

\subsection{3-D image and scattering phase function of a hexagonal particle}

Here, the scattering phase function of a hexagonal ice crystal obtained in experiment HALO02_18 discussed above is presented. To model the scattering phase function produced by such a particle, it is mandatory to define the size, position and orientation of the particle in space with respect to the scattering plane. A 3-D model of a hexagonal ice plate reconstructed from the two images obtained by PHIPS in the HALO02 campaign is shown in Fig. 13 (inset). The dimensions and projections of the three diagonals in each image 


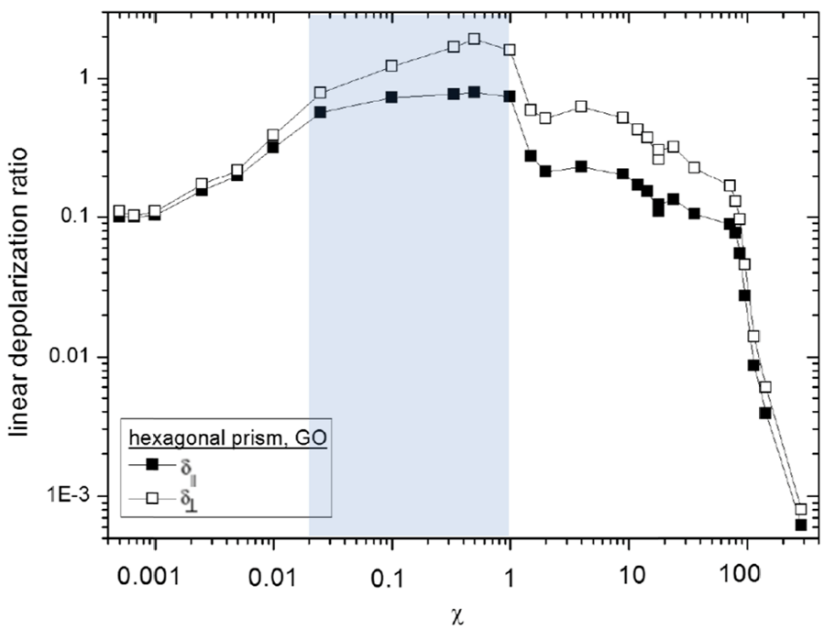

Fig. 12. Linear depolarization ratios vs. $\chi$ of randomly oriented hexagonal prisms calculated using geometric optics. The shaded area represents the wide range of aspect ratios which was deduced from the HOLIMO and PHIPS images throughout HALO02_18 experiment.

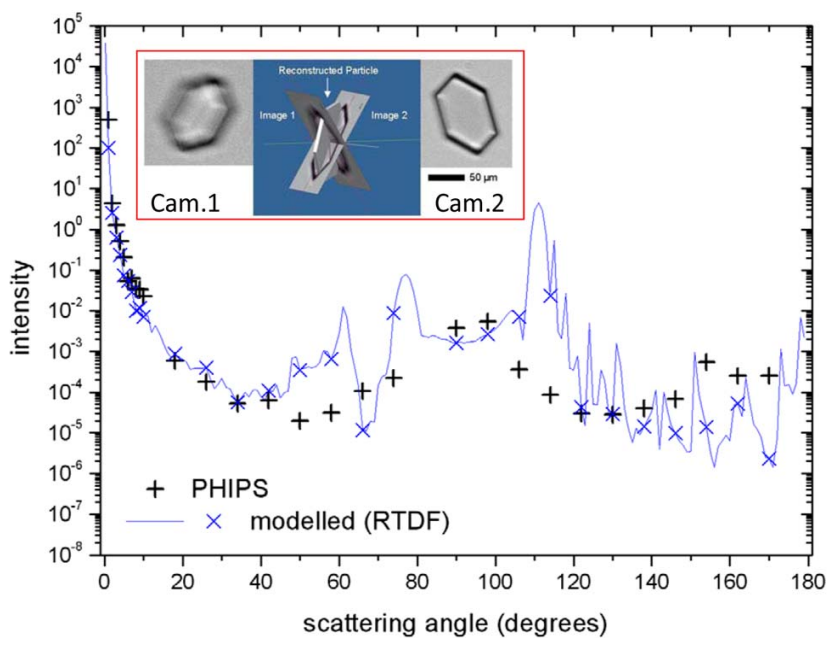

Fig. 13. Comparison between the experimentally obtained polar scattered signal and the one modeled using RTDF for a hexagonal ice crystal observed during the HALO02 campaign. The inset shows the two images collected for the crystal and the reconstructed 3-D model.

were calculated with respect to the horizontal and vertical sides of the image. These inputs were fed into the equations described in Sect. 4.1.6. Referring to the definitions there, it was found that $\alpha=4.63^{\circ}, \beta=38.0^{\circ}$ and $\gamma=27.13^{\circ}$. The dimensions of the constructed hexagonal model are given in Fig. 13. This figure shows a comparison between the experimentally obtained polar scattering signals and, correspondingly, $p 11+p 12$ modeled using RTDF $\left(6 \times 10^{9}\right.$ rays $)$. The polar scattering signal has been investigated for small variations of the azimuthal angle around $\phi=0^{\circ}$, and it has been

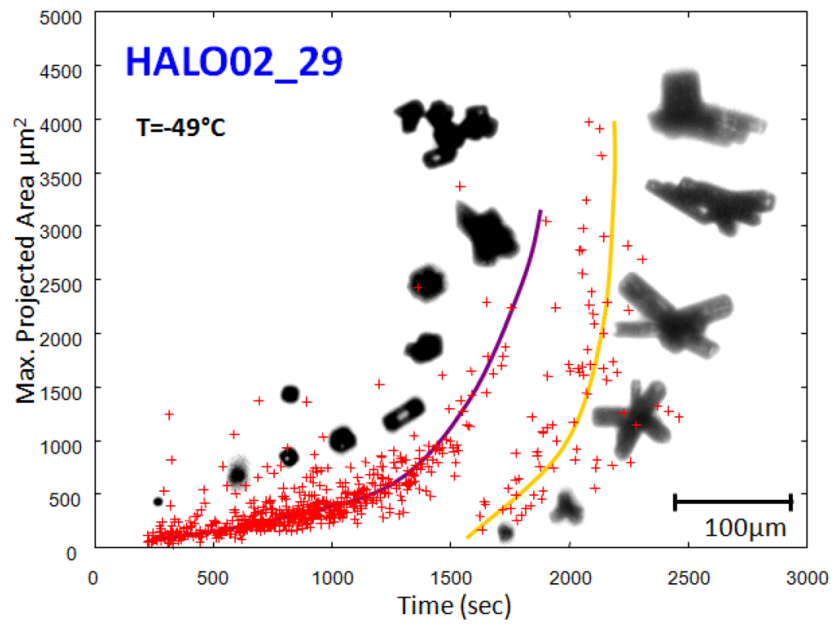

Fig. 14. Different ice nucleation paths depicted by PHIPS during an AIDA expansion (HALO02_29 at $-49^{\circ} \mathrm{C}$ ). The lines are just for guidance to indicate two distinct nucleation and growth events.

confirmed that the standard deviation between measured and calculated intensities for scattering angles larger than $5^{\circ}$ is smallest for $\phi=0^{\circ}$. For the azimuthal angle under investigation, RTDF modeling results give $p 12 \ll p 11$. Therefore, the modeled graph resembles the phase function very closely. The discrepancy between the experimental and modeled results in some angular regions might be due to alignment errors, deviations from the pristine crystal shape and a possible surface roughness. Surface roughness, rounded edges and possibly inclusions might reduce the intensity of the peaks modeled at $62^{\circ}, 78^{\circ}$ and $115^{\circ}$, which can be attributed to rays undergoing multiple internal reflections between the basal facets and less frequent internal reflections at prism facets.

\subsection{HALO02 29 experiment}

The HALO02_29 experiment was conducted similarly to the previously discussed HALO02_18 experiment, but at a lower initial temperature of $-49^{\circ} \mathrm{C}$. Seed ice particles were injected for a short period of a few seconds only at the beginning of the experiment. These ice particles subsequently grew in the chamber maintained under low icesupersaturated conditions with a maximum ice saturation ratio of $s_{i}=1.1$. During this growth period, compact irregular ice particles were detected by PHIPS as depicted in Fig. 14. At about $1000 \mathrm{~s}$ experiment time, the cooling rate was increased to initiate a faster particle growth at higher supersaturation, which resulted in the formation of more complex and distorted ice particle structures. During this fast growth period, the ice particle number concentration continuously decreased due to sedimentation losses which further accelerated the increase in saturation ratio. Eventually, the liquid water saturation ratio was reached at about $1600 \mathrm{~s}$ experiment time when the temperature in the chamber had already 
dropped to about $-57{ }^{\circ} \mathrm{C}$. This resulted in the condensation freezing of the background aerosol particles which were present as interstitial aerosol at a low number concentration of about $1 \mathrm{~cm}^{-3}$ throughout the experiment. This freezing process could be detected clearly by PHIPS as a distinct second growth trace in Fig. 14. The nucleation process under water-saturated conditions obviously resulted in the formation of distinct ice particle structures appearing as four-arm and six-arm rosettes in the PHIPS images. We are not going to dig deeply into the interpretation of these phenomena, since our interest in this paper is to demonstrate the ability of the novel technique to provide unique results.

\subsection{ACI_03_23 experiment}

As PHIPS has also been developed for operation on aircraft, it is reasonable to link its size measurements to data obtained by other airborne instruments. One instrument operated during the AIDA campaigns HALO02 and ACI03 was the Cloud, Aerosol and Precipitation Spectrometer CAPS. Its total number and size distribution concentration measurements are based on two different sub-instruments: the CAS forward scattering and the CIP optical imaging measurements (Baumgardner et al., 2001).

Figure 15 displays the time evolution of the particle sizes measured by CAPS and PHIPS for the ice nucleation experiment ACI03_23. Flame soot particles from a propane diffusion burner (miniCAST, Jing-CAST Technology GmbH, Switzerland) were used as nuclei for the deposition freezing in the AIDA cloud expansion experiment ACI03_23 performed at an initial temperature of $-38.5^{\circ} \mathrm{C}$. In these AIDA studies it was shown that the ice crystal growth deduced from PHIPS in case of small and compact ice particles is in good agreement with retrieval results from in situ FTIR measurements (Schön et al., 2011).

The CAPS is a combination probe. The size measurements of the sub-instruments CAS and CIP are displayed in green and blue in Fig. 15. The PHIPS size measurements are indicated in black. The measurement techniques of the two CAPS subparts are different. CAS measures the optical volume equivalent diameter, whereas CIP detects the area equivalent diameter for each particle. Due to its small sample volume, PHIPS detects only a few particles per second. The small sample volume is necessary when PHIPS is used in the scattering-imaging synchronization mode as mentioned in Sect. 2.3. Due to the larger sample volumes, CAS and CIP detect a larger number of particles per second. For comparison, the median volume diameters of CAS and CIP, calculated from the one second size distributions, are displayed in Fig. 15. The agreement of the particle sizes measured by CAS, CIP and PHIPS is remarkably good in the overlapping size range, especially when considering the different detection methods of PHIPS and CAPS.

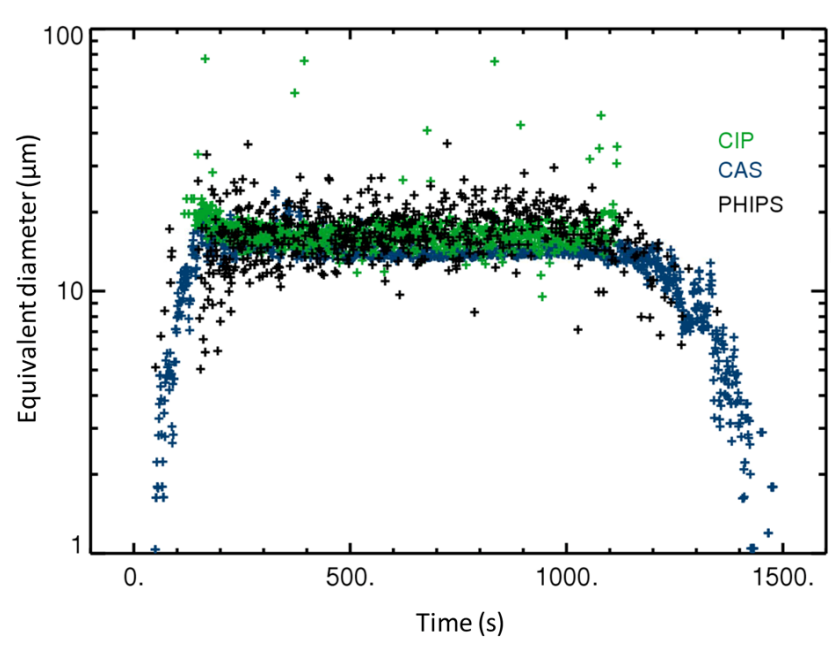

Fig. 15. The time evolution of the median volume diameter obtained from CAPS measurements (CAS (green) and CIP (blue)) and area equivalent diameter from PHIPS measurements (black), for experiment ACI03_23.

\section{Summary and outlook}

The newly developed optical sensor PHIPS is able to simultaneously measure the 3-D morphology represented in the stereo-image and the corresponding optical and microphysical parameters represented in the polar scattering function of individual cloud particles. These two complementary measurements provide the missing correlation between the cloud particle habit and its scattering properties which are used in radiative transfer models. The particle detector of PHIPS ensures that only those particles are captured, which are located in the field-of-view $\times$ depth-of-field volume of the microscope unit. Particles ranging from a few micrometers to about $800 \mu \mathrm{m}$ diameter in size were detected with a maximum scattering acquisition rate of $262 \mathrm{kHz}$ and imaging acquisition rate of $10 \mathrm{~Hz}$. The optical resolving power was $2 \mu \mathrm{m}$ for stereo imaging and the angular resolving power was $1^{\circ}$ in the forward scattering directions (from $1^{\circ}$ to $10^{\circ}$ ) and $8^{\circ}$ in the side and backscattering directions (from $18^{\circ}$ to $170^{\circ}$ ) for the polar scattering. The imaging quality of PHIPS was improved by two different means: (1) the use of a pulsed incoherent illumination laser instead of a flash lamp to illuminate cloud particles enhanced the image quality regarding the background light and the edge sharpness without suffering from diffraction patterns and (2) the inflection point detection algorithm increased the practical depthof-field of the imaging system from $300 \mu \mathrm{m}$ to $1000 \mu \mathrm{m}$. The overall imaging quality was tested in two ways. First, the dependence of size evaluation on particle size for small particles was characterized using standard ice analogues and found to be negligible in the size range from 9.4 to $187.7 \mu \mathrm{m}$. Second, the influence of particle displacement from the focal plane on the investigated particle size was tested using 
an auto scanning-and-imaging system. It showed negligible variances in the investigated particle size for water droplets of $\sim 94 \mu \mathrm{m}$ diameter imaged at different displacements from the focal plane ranging from $-500 \mu \mathrm{m}$ to $500 \mu \mathrm{m}$ along the optical axis of the imaging system. A complete mathematical description was presented to construct a 3-D model of regular geometric shapes like rods, columns and plates from two projection images. The construction of a 3-D model of a hexagonal ice plate from two images was demonstrated, including a comparison between the measured and modeled scattering phase function. A specific particle classification algorithm was introduced to effectively classify particles according to their habits. Finally, the instrument was operated in a series of experiments in the AIDA cloud simulation chamber in the temperature range from $25^{\circ}$ to $-90^{\circ}$ during homogeneous and non-homogeneous temperature-dependent ice crystal growth studies. A comprehensive discussion of one of these experiments was presented, which aimed at improving our knowledge of the relation between ice crystal habits and their ability to scatter and depolarize light. In conclusion, PHIPS proved to be a novel optical sensor which can help to establish the link between the microphysics of cirrus clouds and the single scattering properties of their cloud particles.

The transfer process of PHIPS into PHIPS-HALO has already started and its stereo imaging part is finished and tested. The mechanical part of the polar scattering detection is also finished. The next step is to set up the optical and electrical connections and program the electronic cards of the scattering part. A big challenge is to set the synchronization of both detection parts to handle the detection of high-speed particles $\left(200 \mathrm{~m} \mathrm{~s}^{-1}\right)$, which is the expected speed of the German research aircraft HALO that is planned to carry PHIPSHALO. External housing design and aerodynamic studies are needed to meet the requirements of HALO.

Acknowledgements. We express our gratitude to the technical and scientific staff of IMK-AAF for their continuous support until the achievement of the presented work goals. The work of one of the authors (Evelyn Hesse) was a result of the cooperation with Zbigniew Ulanowski and supported by the National Environment Research Council, UK. This work was funded within the Helmholtz Research Program Atmosphere and Climate and partly by the German Research Foundation (DFG contract SCHN 1140/1-1) within the framework of the priority program 1294. We acknowledge support by Deutsche Forschungsgemeinschaft and Open Access Publishing Fund of Karlsruhe Institute of Technology.

Edited by: S. Schmidt

\section{References}

Amsler, P., Stetzer, O., Schnaiter, M., Hesse, E., Benz, S., Möhler, O., and Lohmann, U.: Ice crystal habits from cloud chamber studies obtained by in-line holographic microscopy related to depolarization measurements, Appl. Optics, 48, 5811-5822, 2009.

Baum, B. A., Yang, P., Hu, Y. X., and Feng, Q.: The impact of ice particle roughness on the scattering phase matrix, J. Quant Spectrosc. Ra., 111, 2534-2549, 2010.

Baumgardner, D., Jonsson, H., Dawson, W., O'Connor, D., and Newton, R.: The cloud, aerosol and precipitation spectrometer: A new instrument for cloud investigations, Atmos. Res., 59-60, 251-264, 2001.

Clarke, A. J. M., Hesse, E., Ulanowski, Z., Kaye, P. H.: A 3D implementation of ray tracing combined with diffraction on facets: Verification and a potential application., J. Quant. Spectrosc. Ra., 100, 103-114, 2006.

Gayet, J. F., Crépel, O., Fournol, J. F., and Oshchepkov, S.: A new airborne polar Nephelometer for the measurements of optical and microphysical cloud properties, Part I: Theoretical design, Ann. Geophys., 15, 451-459, 1997, http://www.ann-geophys.net/15/451/1997/.

Hesse, E.: Modelling diffraction during ray-tracing using the concept of energy flow lines, J. Quant. Spectrosc. Ra., 109, 13741383, 2008.

Hirst, E., Kaye, P. H., Greenaway, R. S., Field, P., and Johnson, D. W.: Discrimination of micrometre-sized ice and supercooled droplets in mixed-phase cloud, Atmos. Environ., 35, 3347, 2001.

Jourdan, O., Mioche, G., Garrett, T. J., Schwarzenböck, A., Vidot, J., Xie, Y., Shcherbakov, V., Yang, P., and Gayet, J. F.: Coupling of the microphysical and optical properties of an Arctic nimbostratus cloud during the ASTAR 2004 experiment: Implications for light-scattering modeling, J. Geophys. Res., 115, D23206, doi:10.1029/2010JD014016, 2010.

Kaye, P. H., Hirst, E., Greenaway, R. S., Ulanowski, Z., Hesse, E., DeMott, P. J., Saunders, C., and Connolly, P.: Classifying atmospheric ice crystals by spatial light scattering, Opt. Lett., 33, 1545-1547, 2008.

Lance, S., Brock, C. A., Rogers, D., and Gordon, J. A.: Water droplet calibration of the Cloud Droplet Probe (CDP) and inflight performance in liquid, ice and mixed-phase clouds during ARCPAC, Atmos. Meas. Tech., 3, 1683-1706, doi:10.5194/amt3-1683-2010, 2010.

Lawson, P. R., Baker, B. A., Schmitt, C. G., and Jensen, T. L.: An overview of microphysical properties of Arctic clouds observed in May and July 1998 during FIRE ACE, J. Geophys. Res., 106, 14989-15014, 2001.

Libbrecht, K. G.: The physics of snow crystals, Rep. Prog. Phys., 68, 855-895, 2005.

Macke, A. and Mishchenko, M. I.: Applicability of regular particle shapes in light scattering calculations for atmospheric ice particles, Appl. Optics, 35, 4291-4296, 1996.

Möhler, O., Stetzer, O., Schaefers, S., Linke, C., Schnaiter, M., Tiede, R., Saathoff, H., Krämer, M., Mangold, A., Budz, P., Zink, P., Schreiner, J., Mauersberger, K., Haag, W., Kärcher, B., and Schurath, U.: Experimental investigation of homogeneous freezing of sulphuric acid particles in the aerosol chamber AIDA, Atmos. Chem. Phys., 3, 211-223, doi:10.5194/acp-3-211-2003, 2003. 
Schnaiter, M., Büttner, S., Vragel, M., and Wagner R.: Influence of particle size and shape on the backscattering linear depolarization ratio of small ice crystals, to be submitted to J. Atmos. Chem. Phys. Discuss., 2011.

Schön, R., Schnaiter, M., Ulanowski, Z., Schmitt, C., Benz, S., Möhler, O., Vogt, S., Wagner, R., and Schurath, U.: Particle habit imaging using incoherent light: a first step towards a novel instrument for cloud microphysics, J. Atmos. Ocean. Tech., 28, 493-512, 2011.

Shcherbakov, V., Gayet, J.-F., Baker, B., and Lawson, P.: Light Scattering by Single Natural Ice Crystals, Am. Meteorol. Soc., 63, 1513-1525, 2006a.

Shcherbakov, V., Gayet, J.-F., Jourdan, O., Ström, J., and Minikin, A.: Light scattering by single ice crystals of cirrus clouds, Geophys. Res. Lett., 33, L15809, doi:10.1029/2006GL026055, $2006 b$.
Takano, Y. and Liou, K. N.: Radiation transfer in cirrus clouds, Part III: light scattering by irregular ice crystals, J. Atmos. Sci., 52, 818-837, 1995.

Ulanowski, Z. J., Hesse, E., Kaye, P. H., Baran, A. J., and Chandrasekhar, R.: Scattering 29 of light from atmospheric ice analogues, J. Quant. Spectrosc. Ra., 79-80, 1091-1102, 2003.

Wagner, R., Linke, C., Naumann, K.-H., Schnaiter, M., Vragel, M., Gangl, M., and Horvath, H.: A review of optical measurements at the aerosol and cloud chamber AIDA, J. Quant. Spectrosc. Ra., 110, 930-949, 2009.

Yang, P., Liou, K. N., Wyser, K., and Mitchell, D.: Parameterization of the scattering and absorption properties of individual ice crystals, J. Geophys. Res., 105, 4699-4718, 2000. 